\title{
Reducing sedated lumbar punctures in pediatric patients with acute lymphoblastic leukemia
}

\author{
Torin Waters ${ }^{1}$ and David Dickens ${ }^{1}$ \\ ${ }^{1}$ University of Iowa
}

June 29, 2021

\begin{abstract}
Background: Sedation for lumbar punctures (LPs) in pediatric acute lymphoblastic leukemia (ALL) patients has been the standard for decades to reduce pain and anxiety. Recent studies on the potential long-term neurocognitive effects of cumulative propofol exposure has raised concerns about this practice. The recent pandemic introduced additional burdens to patients, with the requirement of a negative COVID-19 test prior to each sedated procedure. Procedure: These factors prompted a quality improvement intervention at our institution where we aimed to reduce post-Induction sedated lumbar punctures (LPs) by $50 \%$. Our intervention included patient and family education followed by a simulation of the procedure for selected patients. Those converted to unsedated LPs were queried for their preference. Comparative cost, clinical time and LP success rates were collected for sedated and unsedated LPs. Results: Following the intervention, the percentage of LPs performed with sedation dropped from $100 \%$ to $48.1 \%$. All LPs were successful using both techniques. Most patients who experienced the unsedated LP technique, and their guardians, strongly preferred this approach. Unsedated LPs significantly reduced clinical time (169 vs 83 minutes) for families, decreased expenditures (\$5,736.16 reduction per procedure) and improved institutional opportunity cost due to a decrease in last-minute cancellations. Conclusion: We have shown that it is feasible to significantly reduce the use of sedation for LPs in patients with ALL, which has the potential to improve health and patient experience at a lower cost.
\end{abstract}

\section{Introduction}

The outcomes for children with acute lymphoblastic leukemia (ALL) have improved greatly over the past five decades, with five-year overall survival rates currently around $90 \% .{ }^{1}$ As survival rates improve, attention toward minimizing the late effects of treatment has increased. Performing painful procedures such as lumbar punctures (LPs) under sedation has become routine at most pediatric cancer centers. A recent study showed that among 64 Children's Oncology Group (COG) institutions, >95\% of patients with ALL received LPs with sedation. Propofol was by far the most used sedating agent, with "propofol alone" being the most commonly used sedation regimen (56\% of institutions), and "propofol with opioid and/or midazolam" being used in an additional $31 \%$ of institutions. ${ }^{2}$ Although previous studies have documented that procedural sedation with propofol provides short-term safe and effective control of pain and anxiety ${ }^{3}$, long-term consequences are just beginning to be understood. In December 2016, the US Food and Drug Administration published a warning that repeated or lengthy use of general anesthetic and sedation drugs during surgeries or procedures in children may affect a child's brain development. ${ }^{4}$ In corroboration of these concerns, a recent study of over 200 survivors of childhood leukemia revealed a $40 \%$ increased risk of neurocognitive impairment in patients who received high cumulative doses of propofol sedation. ${ }^{5}$

Contemporary COG protocols for the treatment of ALL involve a minimum of 20 LPs. This number is higher for patients with central nervous system involvement or relapsed disease. Even then, this number does not account for the entire sedation burden these patients experience as they also often require sedation for other procedures such as port placement, bone marrow biopsies, and radiographic imaging. In addition to the risk of neurocognitive deficits, sedated procedures impose a significant financial burden on hospitals and patients, 
due to the involvement of the anesthesia team, use of procedure rooms, and a high frequency of cancelled procedures (low blood counts, respiratory infections, violation of nothing by mouth (NPO) policies, etc.).

The COVID-19 pandemic necessitated many changes to the way our medical systems operate. Starting in March 2020, a negative COVID-19 test has been required within 24 hours of sedated procedures at our institution. Prior to the availability of rapid testing, this necessitated an on-site hospital visit the day before the procedure which generated additional burdens for families, particularly those living with geographic or financial disparity. Due to the medical and operational challenges associated with sedated LPs, a quality improvement project was designed to reduce the number of LPs performed with sedation on ALL patients at our institution.

\section{Materials and Methods}

The following activities were reviewed by the University of Iowa Institutional Review Board and deemed a quality improvement project as they were designed to implement processes that will improve patient care at our program and may not be generalizable due to variation in institutional resources.

\subsection{Setting/Population}

Starting in March 2020, pediatric ALL patients treated at the University of Iowa Stead Family Children's Hospital were given the option to have LPs with or without sedation. This was offered to most patients and families, regardless of age, with a few notable exceptions: patients with an underlying condition such as anxiety or ADHD that might affect their ability to remain calm and still during the procedure, patients in Induction therapy (to reduce the risk of diagnostic LP blood contamination, medication-induced behavioral challenges, and coincidental bone marrow procedures), and patients with anticipated anatomic challenges due to body habitus or a history of multiple attempts with sedated LPs. Two patients with T-lymphoblastic lymphoma were also included in this study due to the similarity of treatment. Sedated and unsedated protocols utilized the same safety checklist protocols ensuring patient identification, consent, and chemotherapy verification. If vincristine was to be delivered on the same day as an unsedated procedure, the vincristine (always dispensed in a mini-infuser bag) was delivered and completed prior to the start of the procedure.

\subsection{Intervention}

At an appointment leading up to an LP, the entire procedure was explained to the patient (when ageappropriate) and guardian. If the patient and family agreed to consider an unsedated LP, a simulation including positioning and palpation of landmarks was performed. If the patient was able to remain still throughout the simulation, the upcoming LP was scheduled without sedation.

All patients had topical lidocaine applied to the lumbar area upon arrival to clinic. Most patients received a dose of an anxiolytic medication, either lorazepam $(0.05 \mathrm{mg} / \mathrm{kg} /$ dose PO/IV, max dose $2 \mathrm{mg})$ or midazolam $(0.2 \mathrm{mg} / \mathrm{kg}$ PO, max dose $20 \mathrm{mg}) 30$ minutes prior to the LP. The guardian was given the option to be present with the patient in the room along with a Child Life team member to aid in keeping the patient distracted and calm. Buffered lidocaine was used according to the discretion of the provider performing the procedure. Unsedated LPs were performed in a clinic or procedure room in the Pediatric Hematology/Oncology clinic, whereas patients requiring sedated LPs were transported to another floor with procedure rooms and pre- and post-anesthesia recovery rooms. Sedated and unsedated LPs were performed by pediatric oncology faculty, fellows, and advanced practice providers.

\subsection{Aims}

Our primary aim was to decrease the number of post-Induction sedated LPs performed in pediatric patients with ALL at the University of Iowa by $50 \%$. Our secondary aims were to compare sedated and unsedated LPs with regards to patient/caregiver preferences, time spent in clinic, percentage of successful and bloodcontaminated LPs, and overall costs, and to perform value stream mapping to improve efficiency of the LP process.

\subsection{Measures}




\subsubsection{Quantifying Sedated and Unsedated Lumbar Punctures}

The intervention period lasted for approximately seven months (3/2020-10/2020), during which time we educated staff, performed the visual process mapping, and refined our policies and procedures for unsedated LPs. The electronic medical records of all pediatric patients with ALL undergoing active treatment at the University of Iowa during the post-intervention period (10/1/2020-12/31/2020) were retrospectively reviewed and the numbers of sedated and unsedated LPs for each patient were recorded. For this patient cohort, the same data was recorded from an equivalent defined pre-intervention period (10/1/2019 to 12/31/2019), if those patients were undergoing treatment at that time.

\subsubsection{Observations and Process Mapping}

Our quality improvement intervention utilized a value stream mapping approach to identify efficiency gaps in our institution's sedated and unsedated LP processes. Members of the University of Iowa Quality Improvement Program helped create process maps for sedated and unsedated LPs after performing observations of clinic visits for patients receiving both types of procedures to quantify the time utilized for each step of the process. These steps were characterized as "value added" (directly relating to patient care) and "non-value added" (not relating to patient care, such as waiting time, transportation, etc.).

\subsubsection{CSF Characteristics}

To evaluate the quality of cerebrospinal fluid (CSF) samples obtained during both types of procedures, data on the number of red blood cells were retrospectively reviewed.

\subsubsection{Sedation Preference Survey}

Patient guardians, as well as patients over the age of 7 , were given a two-question survey to assess their preference between LPs with and without sedation. The survey was developed by the authors, then tested and revised according to feedback from members of the nursing and quality team prior to dissemination. The surveys were introduced and handed out to guardians and age-appropriate patients by nursing staff and returned the same day. The two questions and response options were, "Please indicate your overall preference for sedated versus unsedated LPs on a scale from 0 to 10. A rating of 0 indicates you strongly prefer LPs with sedation and a 10 means you strongly prefer LPs without sedation." and "How much do the current COVID-19 swab requirements influence your answer to the previous question? Unsure/Not at all/A little/A lot".

\subsubsection{Costs}

Overall charges associated with the patient visit on procedure days with and without sedation were tabulated and compared. The number of cancelled procedures during the post-intervention period was collected.

\section{Results}

Our goal of reducing the number of post-Induction sedated LPs by $50 \%$ in pediatric ALL patients was met. During the three-month post-intervention period from 10/1/20 to 12/31/20, 37 out of 77 post-Induction LPs were performed with sedation (48.1\%) compared to 59 out of $59(100 \%)$ in the same patient group one year earlier (10/1/19 to 12/31/19) (Fig. 1). Inclusive of Induction LPs, the total number of LPs in the post-intervention period was 91 . Additional patient information is shown in Table 1.

Process maps developed for both techniques demonstrated that unsedated LPs provided a more streamlined approach, involving 42 total steps instead of 53, and 6 non value-added steps versus 16 . Similarly, observations of clinic visits for patients receiving sedated or unsedated LPs revealed substantial differences in clinic visit duration. Six sedated LP visits and five unsedated LP visits were observed. Clinical time (visit time related directly to patient care, excluding wait and transportation times) was, on average, more than twice as long (169 minutes compared to 83 minutes) for patients receiving sedated LPs instead of unsedated, primarily related to involvement of the anesthesia team (evaluation and recovery). 
An additional factor related to optimizing the LP process for both the institution and for patients is the cancelling of sedated procedures. During the post-intervention period, $30 \%$ of scheduled sedated LPs were cancelled for reasons such as NPO violations, positive COVID-19 tests, and viral upper respiratory infections. Patients receiving unsedated LPs were not tested for COVID-19 and were able to proceed with their procedures if symptoms of mild respiratory infection were present.

Analysis of CSF characteristics obtained from LPs during the post-intervention period revealed a higher incidence of blood in the CSF ( $>500 \mathrm{RBCs}$ ) for unsedated procedures $(5 / 43,11.6 \%)$ as compared to sedated procedures $(0 / 48,0 \%)$ (Fig. 2$)$. There were no failed LPs in either group.

Surveys were provided to 19 patients who received both types of LPs, and 16 were returned (response rate $84.2 \%$ ). Patient and guardian preferences for unsedated vs sedated LPs (evaluated on a 0 to 10 scale, 10 indicating a strong preference for unsedated LPs) showed a mean response of 9.3 for guardians and 8.5 for patients (Fig. 3). $43 \%$ of guardians and $33 \%$ of patients indicated that COVID-19 testing requirements did not play a significant role in their responses (Fig. 4), suggesting that some might choose to continue with unsedated LPs even if COVID-19 testing was not required.

Finally, costs were approximated by comparing the overall charges to patients for sedated and unsedated LPs, and by assessing the cumulative opportunity cost of cancelled LPs. The average overall charges to a pediatric patient with ALL at the University of Iowa for a clinic visit involving a sedated LP, inclusive of labs, medications, involvement of the anesthesia team and use of recovery rooms, total $\$ 10,620.85$ (average obtained from 6 observed sedated LPs). For a visit with an unsedated LP, the average overall charges total $\$ 4,884.69$ (average obtained from 5 observed unsedated LPs). This results in a cost reduction of $\$ 5,736.16$ per procedure. Extrapolating the total number of LPs performed during our post-intervention window, 91, to an entire year, provides an estimate of 364 LPs per year. If $50 \%$ of these are performed unsedated, there is an approximate health care expenditure reduction of $\$ 1,043,981$ to pediatric patients with ALL per year. During the post-intervention period, 21 sedated LPs were cancelled. The average usage time of sedated procedure and recovery rooms per sedated LP was determined to be 91 minutes, and the total charges, including anesthesia team and recovery rooms, approximately $\$ 15,392.54$. Extrapolating these numbers results in an opportunity cost of 128 hours and $\$ 1,290,000$ per year to our institution.

\section{Discussion}

As pediatric cancer outcomes have improved over the decades, focus has shifted toward avoiding harmful side effects of treatment. This is especially true of cancers with high rates of survival, such as pediatric ALL. While procedural sedation has been routinely utilized for LPs in pediatric patients with ALL for decades, emerging evidence regarding the deleterious neurocognitive effects of repeated propofol exposure raises concerns about what is best for the long-term health of the patient.

The COVID-19 pandemic brought many challenges to health care institutions, but also provided opportunities for reevaluation and optimization of many processes. The burden of clinic visits on consecutive days for COVID-19 testing prior to procedures became yet another stressor for families already contending with the complex care of a child with leukemia.

At our institution, we began offering unsedated LPs to most of our pediatric patients with ALL and were able to achieve our goal of reducing the number of post-Induction sedated LPs by over $50 \%$. Secondarily, we found that unsedated LPs improved the patient/family experience and reduced expenditures while also improving cost opportunity. Most importantly, the conversion to unsedated LPs could potentially address one of the significant late effects of ALL treatment, neurocognitive decline. Further studies aimed specifically at comparing neurocognition in ALL survivors treated with unsedated vs sedated LPs are needed to support this hypothesis.

One potential problem observed for our unsedated LPs was an increased incidence of CSF samples containing blood. Importantly, there were no failed procedures with the unsedated approach. Of the five bloodcontaining CSF specimens, three came from a single patient who was later converted back to sedated LPs 
and continued to have blood-containing specimens on occasion. Another came from a child who previously had blood in a sedated CSF sample. While there are no known risks of introducing blood into the CSF for patients with ALL in remission, inferior outcomes of patients with traumatic LPs at diagnosis are welldescribed. ${ }^{6,7}$ The increased incidence of traumatic LPs without sedation supports our reasoning to avoid this technique at diagnosis. Given this experience, additional proven strategies to improve LP techniques such as ultrasound guidance are being explored at our institution to enhance technique efficiency ${ }^{8}$.

With regards to patient and family preferences, the majority responded very strongly in favor of the unsedated approach. A significant percentage of patients and families indicated that mandatory COVID-19 testing did not strongly influence this preference. Other benefits of unsedated LPs noted by patients and families include lack of NPO requirements, shorter hospital visit time, and allowance of caregiver presence in the room during the procedure. While not the motivating force behind our study, our analyses also suggest a potentially significant financial benefit of unsedated LPs, both to payers and treating institutions. An estimated decrease in patient charges of $\$ 5,700$ per procedure visit is substantial considering they will receive at least 20 during their therapy. Last-minute cancellations for sedated LPs in ALL patients are a regular part of practice that create a burden for institutional sedation services. In our study, during the 3-month post intervention period, $30 \%$ of sedated LPs were cancelled with 24 hours of the procedure, providing inadequate time to utilize the scheduled sedation resources to maximal capacity. Depending on the size of the treating institution, decreasing opportunity cost and improving access for all patients in need of sedation could be substantial benefits associated with the intervention described in this report.

Our results are assumed to be generalizable, as unique institutional and contextual factors during the intervention period may have affected our findings. Fewer patients may have been willing to convert to unsedated LPs without a COVID-19 testing mandate, and the requirement of hospital visits on consecutive days may have been less problematic at institutions with a smaller geographic catchment area. In addition, the individualized approach to patient/family counseling as well as the significant procedural experience among our medical staff may not be available at some institutions.

Our findings suggest that performing LPs without sedation in pediatric patients with ALL offers several key benefits, including improving the patient and family experience as well as decreasing health care expenditures. This is in addition to prior evidence that minimizing exposure to propofol sedation could reduce long-term neurocognitive side effects. Increasing the number of unsedated LPs performed in pediatric patients with ALL provides an opportunity to deliver better care in a more streamlined, cost-effective manner, warranting consideration of implementing such a strategy on a wider scale.

\section{Conflict of Interest:}

The authors declare that there is no conflict of interest.

\section{Data Availability Statement:}

The data that support the findings of this study are available on request from the corresponding author. The data are not publicly available due to privacy or ethical restrictions.

\section{References:}

1. Hunger SP, Mullighan CG. Acute Lymphoblastic Leukemia in Children. N Engl J Med. 2015;373(16):15411552 .

2. Nugent BD, Davis PJ, Noll RB, Tersak JM. Sedation practices in pediatric patients with acute lymphoblastic leukemia. Pediatr Blood Cancer. 2020;67(2):e28037.

3. Aslam SL, Haque A, Jamil MT, Ariff M, Nasir S. Safety and Efficacy of Procedural Sedation and Analgesia in Pediatric Oncology Patients. Cureus. 2020;12(3):e7442.

4. U.S. Food and Drug Administration (FDA). FDA review results in new

warnings about using general anesthetics and sedation drugs in young children and pregnant women. 2016. 
5. Banerjee P, Rossi MG, Anghelescu DL, et al. Association Between Anesthesia Exposure and Neurocognitive and Neuroimaging Outcomes in Long-term Survivors of Childhood Acute Lymphoblastic Leukemia.JAMA Oncol. 2019;5(10).

6. Shaikh F, Voicu L, Tole S, et al. The risk of traumatic lumbar punctures in children with acute lymphoblastic leukaemia. Eur J Cancer. 2014;50(8):1482-1489.

7. Gajjar A, Harrison PL, Sandlund JT, et al. Traumatic lumbar puncture at diagnosis adversely affects outcome in childhood acute lymphoblastic leukemia. Blood. 2000;96(10):3381-3384.

8. Shaikh F, Arzola C, Alexander S, et al. Feasibility of ultrasound-assisted lumbar punctures performed by pediatric oncologists at the point of care. Pediatr Blood Cancer. 2021;68(7):e29015.

\section{Legend}

Figure 1. Number of sedated vs unsedated LPs in pediatric patients with ALL performed during the pre-intervention period $(10 / 1 / 2019-12 / 31 / 2019)$ compared to the post-intervention period $(10 / 1 / 2020$ $12 / 31 / 2020)$.

Figure 2. Number of CSF samples containing blood (>500 RBCs) in the post-intervention period (10/1/2020$12 / 31 / 2020)$, for sedated vs. unsedated LPs.

Figure 3. Average survey response scores to regarding preference for unsedated vs sedated LPs from parents and patients. Scale response options ranged from 0 to 10, with 0 indicating a strong preference for sedated LPs, and 10 indicating a strong preference for unsedated LPs.

Figure 4. Survey responses from parents and patients regarding how much COVID-19 testing requirements impacted their preference for sedated vs unsedated LPs.

Table 1: Characteristics of pediatric patients with ALL and T-lymphoblastic lymphoma treated in the threemonth post-intervention period (10/1/2020-12/31/2020). One patient is not included in the section "Type of Lumbar Puncture Received", as that patient only received Induction lumbar punctures during this period.

\section{Hosted file}

Table 1.docx available at https://authorea.com/users/356310/articles/528323-reducing-sedatedlumbar-punctures-in-pediatric-patients-with-acute-lymphoblastic-leukemia 


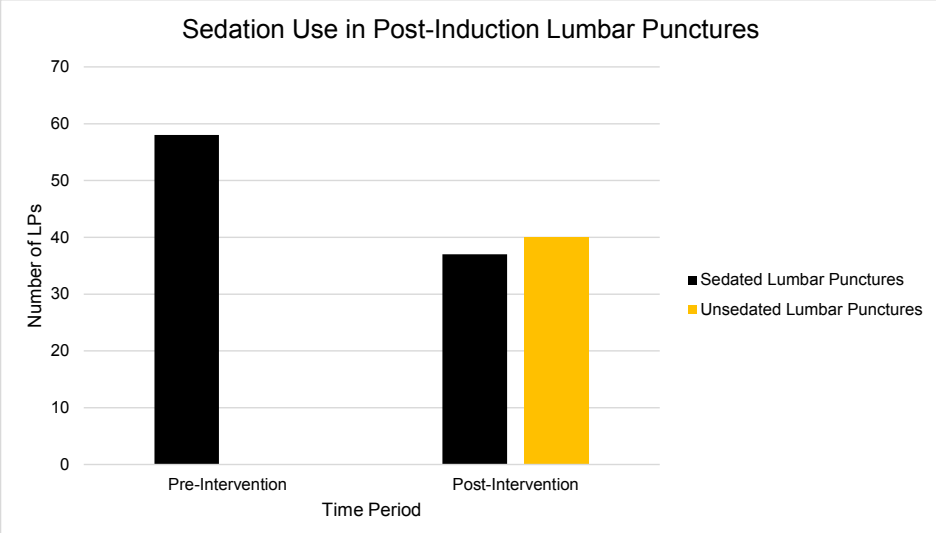




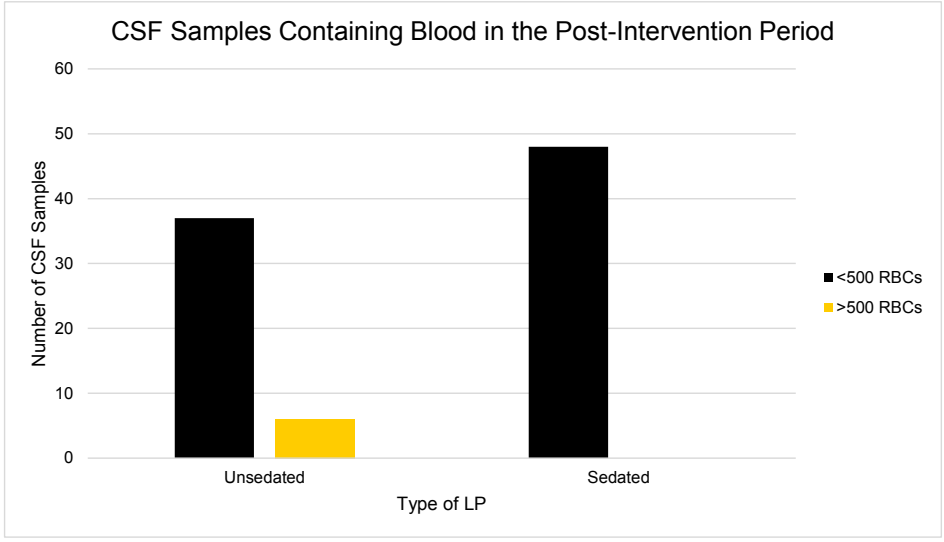




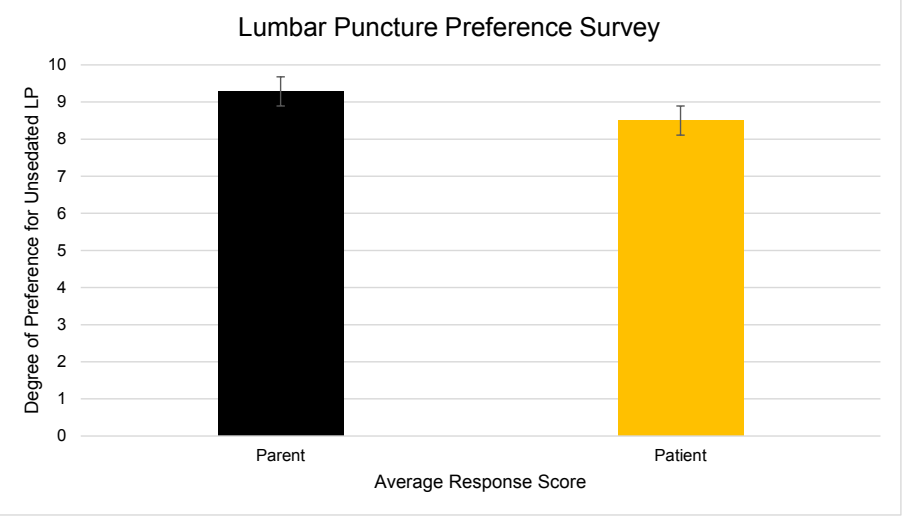




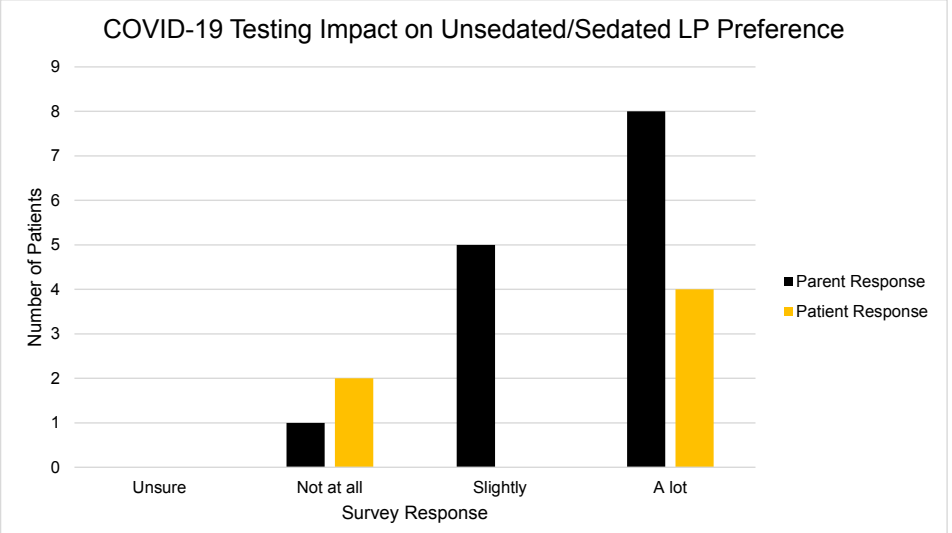

